Research

Open Access

\title{
Sedation practice in the intensive care unit: a UK national survey
} Henrik Reschreiter ${ }^{1}$, Matt Maiden ${ }^{1}$ and Atul Kapila ${ }^{2}$

\author{
${ }^{1}$ Royal Adelaide Hospital, North Terrace, Adelaide, SA 5000, Australia \\ ${ }^{2}$ Royal Berkshire and Battle Hospital NHS Trust, London Road, RG1 5AN, Reading, UK \\ Corresponding author: Henrik Reschreiter, henrik.reschreiter@hotmail.com
}

Received: 6 Oct 2008 Revisions requested: 5 Nov 2008 Revisions received: 27 Nov 2008 Accepted: 1 Dec 2008 Published: 1 Dec 2008

Critical Care 2008, 12:R152 (doi:10.1186/cc7141)

This article is online at: http://ccforum.com/content/12/6/R152

(C) 2008 Reschreiter et al.; licensee BioMed Central Ltd.

This is an open access article distributed under the terms of the Creative Commons Attribution License (http://creativecommons.org/licenses/by/2.0), which permits unrestricted use, distribution, and reproduction in any medium, provided the original work is properly cited.

\begin{abstract}
Introduction The purpose of this study was to evaluate sedation practice in UK intensive care units (ICUs), particularly the implementation of daily sedation holding, written sedation guidelines, sedation scoring tools and choice of agents.

Methods A national postal survey was conducted in all UK ICUs.

Results A total of 192 responses out of 302 addressed units were received (63.5\%). Of the responding ICUs, 88\% used a sedation scoring tool, most frequently the Ramsey Sedation Scale score $(66.4 \%)$. The majority of units have a written

sedation guideline ( $80 \%$ ), and $78 \%$ state that daily sedation holding is practiced. A wide variety of sedating agents is used, with the choice of agent largely determined by the duration of action rather than cost. The most frequently used agents were propofol and alfentanil for short-term sedation; propofol, midazolam and morphine for longer sedation; and propofol for weaning purposes.

Conclusions Most UK ICUs use a sedation guideline and sedation scoring tool. The concept of sedation holding has been implemented in the majority of units, and most ICUs have a written sedation guideline.
\end{abstract}

\section{Introduction}

Patients requiring mechanical ventilation in the intensive care unit (ICU) usually require a sedating agent [1]. Sedation reduces the negative physiological effects of the stress response to mechanical ventilation $[2,3]$ and may reduce the psychological issues patients may face after critical illness [4]. However, excessive sedation may be harmful. Over-sedation can contribute to hypotension, venous thrombosis, prolonged ventilation, an increased risk for pneumonia and a prolonged stay in the ICU, with an increasing burden on staff, bed availability and associated costs $[5,6]$.

Recent evidence indicates that the choice of sedating agents, frequency of administration and regular assessment of sedation contribute to patient outcomes [7-9]. Kress and coworkers [7], in 2000, demonstrated that daily interruption of sedation reduced ventilation duration, ICU length of stay, complications such as venous thromboembolic disease, upper gastrointestinal bleeding and bacteraemia, and the incidence of post-traumatic stress disorder $[10,11]$.
There have been a number of systematic reviews of sedation practice in the ICU and subsequent evidence-based clinical practice guidelines for sedation [12-16]. However, uptake of these evidence-based guidelines is variable. Sedation surveys in a range of countries have demonstrated different practices in the management of sedation [17-21]. The last survey of sedation practice in UK ICUs was published in 2000 [22], before the concept of daily sedation holding was published. The rate of implementation of current sedation guidelines in UK ICUs is unknown.

This UK national survey was performed to assess the impact of published trials and guidelines on ICU sedation practice since 2000.

\section{Materials and methods}

A tick-box questionnaire was developed to survey sedation practice [see Additional data file 1]. The questionnaire was sent to all UK ICUs. The list of units was obtained from the Intensive Care National Audit \& Research Centre and crossreferenced with the Directory of Critical Care 2006 (CMA 
Medical Data, Loughborough). The questionnaire and covering letter was addressed to the 'Clinical Director' of the ICU, and a stamped self-addressed return envelope was provided.

The local ethics committee (Royal Berkshire NHS Foundation Trust, Reading, UK) was approached, but formal processing and approval was deemed unnecessary.

The questionnaire was posted out in November 2006. Those ICUs that did not reply received follow-up questionnaires in December 2006 and March 2007. The last response was received in June 2007.

The data were entered into a database (Microsoft Excel Office 2003; Microsoft Corp., Redmond, WA, USA). The data were then read into version 9.1 of the $S A S^{\circledR 1}$ system (SAS Institute Inc., Cary, NC, USA) running under Microsoft Windows XP, where they were summarized and analyzed. Data were crosstabulated as appropriate and Mantel-Haenszel $\chi^{2}$ tests were used for analysis. Paired $t$-tests were used to look for any differences for cost versus duration of action. Differences were deemed to be statistically significant at $P<0.05$.

\section{Results}

A total of 302 UK ICUs were identified and responses were received from $192(63.5 \%)$. Seven of these responses were excluded from further analysis; five were high dependency units that do not admit ventilated patients, and two questionnaires were returned blank. The denominator used for the results and statistical analysis was 185 . The geographical distribution revealed that 155 hospitals were situated in England, 15 in Scotland, 10 in Wales and five in Northern Ireland. The demographic data of the replying ICUs are outlined in Table 1 and reveal that a wide range of ICUs were surveyed.

Table 2 illustrates that $88.1 \%$ of UK ICUs utilize a sedation scoring tool. The Ramsey Sedation Scale score [23] is the most widely used (66.5\%). A number of ICUs have developed their own sedation scores (details unknown), and have named them after their place of development/workplace.

Most UK ICUs (80\%) have a written sedation guideline and $78 \%$ practice daily sedation holding. However, only $53 \%$ of ICUs audit compliance with their guidelines (Table 2). No difference could be observed between units of different size or depending on number of admissions (Table 3 ).

Neuromuscular blocking agents are infrequently used, with $71 \%$ of ICUs using it less than $5 \%$ of the time. However, $7 \%$ of ICUs use muscular blocking agents in more than $10 \%$ of their patients; these ICUs were predominantly neurosurgical (Table 4).

According to visual-analogue scale assessment $(0=$ not affecting decision and $10=$ main factor), choice of sedating
Table 1

\begin{tabular}{ll} 
General Data & \\
\hline Variable & Number of units $(\%)$ \\
\hline Number of beds & $26(14 \%)$ \\
\hline 0 to 4 & $99(53 \%)$ \\
5 to 8 & $36(19 \%)$ \\
9 to 12 & $24(13 \%)$ \\
$>12$ & Total: 185 units \\
\hline Number of ICU admissions/yeara \\
\hline$<250$ & $17(9 \%)$ \\
250 to 500 & $83(45 \%)$ \\
500 to 750 & $43(23 \%)$ \\
750 to 1,000 & $22(12 \%)$ \\
$>1,000$ & $17(9 \%)$ \\
& Total: 182 units
\end{tabular}

$\%$ ventilated patients ${ }^{a}$

\begin{tabular}{ll}
0 to 25 & $7(4 \%)$ \\
26 to 50 & $45(24 \%)$ \\
51 to 75 & $79(43 \%)$ \\
76 to 100 & $49(26 \%)$ \\
& Total: 180 units \\
\hline
\end{tabular}

\begin{tabular}{ll}
\hline Type of patients $^{\mathrm{b}}$ & \\
\hline Surgical & $157(85 \%)$ \\
Medical & $156(84 \%)$ \\
Cardiac & $13(7 \%)$ \\
Neurological & $21(11 \%)$ \\
\hline
\end{tabular}

aNo answer to the question was given by some units. bMore than one type of patient in an ICU was possible. ICU, intensive care unit

agent is strongly influenced by duration of action. In comparison, cost of the sedating drug has less of an influence on sedation choice (mean visual-analogue scale score cost 4.4 versus duration of action $6.4 ; P<0.0001$; Figure 1 ).

A range of sedating agents are used by the surveyed ICUs (Table 5). Propofol is the most frequently used sedating agent for patients with expected duration of ICU admission less than 24 hours. For an expected ICU admission of more than 24 hours, midazolam and propofol are the most commonly used agents. During ventilator weaning, propofol is used most frequently, with clonidine being the next most commonly used agent. 
Table 2

\section{Sedation scoring practice}

\begin{tabular}{ll}
\hline Question & Number of units (\%) \\
\hline Do you use a sedation score? & $163(88.1 \%)$ \\
Yes & 19 \\
No & \\
\hline Which sedation score do you use? (several answers possible) & $123(66.5 \%)$ \\
Ramsey Sedation Scale & $10(5.4 \%)$ \\
Richmond Agitation Sedation scale & $4(2.1 \%)$ \\
Bispectral Index & $7(3.8 \%)$ \\
Score of the UK Intensive Care Society & $4(2.2 \%)$ \\
Sheffield & $2(1.1 \%)$ \\
Bloomsbury & $2(1.1 \%)$ \\
Cambridge & $3(1.6 \%)$ \\
Cook & $3(1.6 \%)$ \\
Local scoring system, unspecified & $7(3.8 \%)$ \\
Other, but not specified & $3(1.6 \%)$ \\
No answer given & \\
\hline
\end{tabular}

Do you have a sedation guideline?

Yes

$148(80 \%)$

No

37

Do you practice daily sedation holding?

Yes

No

Do you audit your compliance with your sedation holding guideline?

Yes

No

82

If you audit your compliance with your sedation holding practice, what is your compliance?

$0 \%$ to $40 \%$

5

$40 \%$ to $60 \%$

14

$60 \%$ to $80 \%$

26

$80 \%$ to $90 \%$

23

$90 \%$ to $100 \%$

24

The sum of answers is less than 185 in some cases as no answer to the question was given by some units.

A wider range of analgesic agents is used during the sedation of ICU patients. For short-term analgesia, alfentanil, morphine, fentanyl and remifentanil are commonly used. For longer expected sedation ( $>24$ hours), morphine is the most commonly used agent.

\section{Discussion \\ Sedation scoring}

The majority of responding ICUs (88\%) in our survey use a sedation scoring system. This has increased considerably since the UK survey in 2000, when $67 \%$ of hospitals used a scoring system [22]. Despite this increased uptake since the 
Table 3

\begin{tabular}{|c|c|c|c|c|c|}
\hline Number of beds & $\begin{array}{l}\text { Number of ICUs } \\
(n=185)\end{array}$ & $\begin{array}{l}\text { Sedation guideline }(n= \\
148)^{\#}\end{array}$ & No guideline $(n=37)$ & $\begin{array}{l}\text { Daily sedation holding } \\
(n=144)\end{array}$ & $\begin{array}{l}\text { No sedation holding } \\
(\mathrm{n}=41)\end{array}$ \\
\hline 0 to 4 & 26 & $19(73 \%)$ & $7(27 \%)$ & $20(77 \%)$ & $6(23 \%)$ \\
\hline 5 to 8 & 99 & 78 (79\%) & $21(21 \%)$ & 78 (79\%) & $21(21 \%)$ \\
\hline 9 to 12 & 36 & $30(83 \%)$ & $6(17 \%)$ & $31(86 \%)$ & $5(14 \%)$ \\
\hline$>12$ & 24 & $20(83 \%)$ & $3(13 \%)$ & $15(63 \%)$ & $9(37 \%)$ \\
\hline
\end{tabular}

Percentages are out of the ICUs of that size group. No statistically significant differences were observed. \# One ICU with more than 12 beds left this question unanswered. ICU, intensive care unit.

last survey and favourable comparison with other countries, an evidence-based approach is still not universally followed.

Numerous sedation assessment tools have been developed to minimize this risk for over-sedation. Some sedation scales have been validated against other scales in patients (for example, Riker Sedation-Agitation Scale [24,25], Motor Activity Assessment Scale [26], Vancouver Interaction and Calmness Scale [27], and more recently the Richmond Agitation-Sedation Scale [28,29], published after the American College of Critical Care Medicine (ACCM) guidelines [12]. The latter has shown an excellent performance, not only with regard to interrater reliability and validity, but it is also the first score to detect changes over time in the critically ill patient. However, no consensus yet exists in an international guideline regarding which assessment tool to use, and validation itself is problematic because of the lack of a standard to validate against that is not based on opinion.

The uptake of the Richmond Agitation-Sedation Scale has been slow in previous surveys. It did not feature in the 2005 German survey [17] or in the Canadian survey conducted in 2006 [18], with one unit reporting its use in the 2007 German update survey. The French survey in 2007 [21] and our study are the first reports of its use being more widespread.

The sedation scale used most commonly in this survey was the Ramsey Sedation Scale [23], with $66.5 \%$ of ICUs using this sedation assessment. Furthermore, most of the other scales used are adaptations of the Ramsay Sedation Scale (for example, the UK Intensive Care Society sedation scale). The choice of sedation scoring tool has not changed since the last UK survey, with the Ramsay Sedation Scale score being used most commonly then (40 out of 142 units stating that they used a sedation scoring system in 2000) [22].

The widespread use of the Ramsay Sedation Scale is in contrast to the ACCM guidelines [12], which recommend use of the validated assessment scores, such as the Motor Activity Assessment Scale, Riker Sedation-Agitation Scale, and Vancouver Interaction and Calmness Scale. Its advantages appear to be familiarity to staff and simplicity, and it is the scale that has been most commonly used historically despite its clinical limitations. However, the Ramsay Sedation Scale lacks clear discrimination and exhibits considerable inter-rater variability [30].

The practice of sedation assessment in the UK differs from that in other countries. In Germany in 2005 only 51\% of responding ICUs report sedation monitoring, with the Ramsay Sedation Scale used 'almost exclusively'[17], and in Canada in 2006 only $49 \%$ of responding ICUs utilized sedation monitoring, with $69 \%$ of ICUs using the Ramsay Sedation Scale $[17,18,20]$. Our results are consistent with a 2001 sedation survey conducted in European ICUs [28], which showed that ICUs in the UK use sedation scales more frequently than do

Table 4

\begin{tabular}{lll}
\hline \multicolumn{2}{l}{ Neuromuscular blocking agents used } & \\
\hline$\%$ of patients & Number of ICUs & Number of neurological ICUs \\
\hline $0 \%$ to $5 \%$ & $128(71 \%)$ & 4 neurological ICUs \\
$6 \%$ to $10 \%$ & $39(22 \%)$ & 7 neurological ICUs \\
$11 \%$ to $15 \%$ & $6(3 \%)$ & 2 neurological ICUs \\
$16 \%$ to $20 \%$ & $6(3 \%)$ & 5 neurological ICUs \\
$>20 \%$ & $2(1 \%)$ & 1 neurological ICU, one cardiac-ICU
\end{tabular}

The denominator was 181 (four responding ICUs did not answer this question). ICU, intensive care unit. 
Figure 1

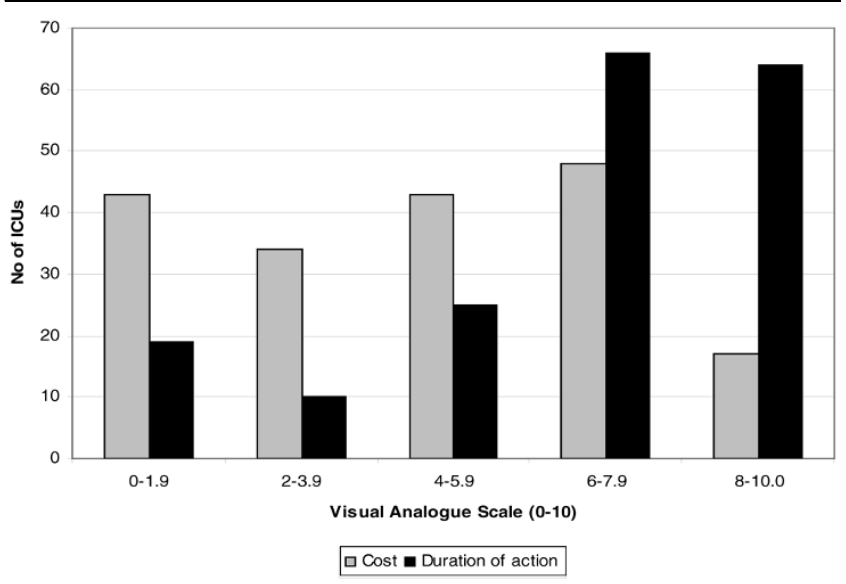

Importance of cost and duration of action on choice of agent. A total of 185 units are included in this analysis. VAS, visual analogue scale (range: $0=$ not important to $10=$ most important).

those in all other participating European countries (72\% of UK ICUs).

\section{Sedation guidelines}

It has been shown in many but not all studies over the past decade that an ICU sedation protocol results in fewer days on the ventilator, a shorter stay in the ICU and reduced costs [3137]. Despite the mostly good evidence and a comparably easy and cheap means of improving care, not all hospitals have implemented a formal sedation guideline.
In this survey, $80 \%$ of the responding hospitals have an operating sedation guideline, which has increased sharply since 2000 , when $43 \%$ of participating hospitals stated that they had a written guideline. A German survey conducted in 2007 [20] revealed that $46 \%$ of hospitals used a sedation guideline, and the Canadian survey in 2006 [18] reported that 29\% of ICUs used a sedation protocol.

Our high rate of ICUs reporting use of a sedation guideline could reflect reporter bias, because units with more interest in sedation may be more likely to respond to this questionnaire. However, there has been increased utilization of sedation guidelines in Germany in the recent years, suggesting that our results may reflect actual change in practice [20].

\section{Sedation holding}

In 2000, Kress and coworkers [7,11] showed that daily withholding of sedative agents led to reduced length of ICU stay, less ventilator time, fewer ICU complications and fewer neurological investigations. Subsequent studies by the same group demonstrated daily sedation withholding to be safe in patients with ischaemic heart disease, and that it reduces the psychological sequelae of critical illness [10,38]. A different group, however, raised safety concerns in a trial including a high percentage of patients (around 30\% to 40\%) with alcohol and other drug use disorders, emphasizing that patient selection and an individualized approach is important [39].

A recent pilot trial addressed the issue of safety and feasibility of daily interruption of sedation with simultaneous use of protocolized sedation [40]. The authors concluded that in their

Table 5

\begin{tabular}{|c|c|c|c|}
\hline Expected length of stay in the ICU & $<24$ hours & $>24$ hours & Weaning \\
\hline \multicolumn{4}{|l|}{ For sedation } \\
\hline Propofol & 181 & 111 & 65 \\
\hline Midazolam & 23 & 136 & 8 \\
\hline Clonidine & 2 & 20 & 22 \\
\hline Lorazepam & 2 & 13 & 3 \\
\hline Haloperidol & 1 & 5 & 7 \\
\hline Diazepam & 1 & 2 & 1 \\
\hline \multicolumn{4}{|l|}{ For analgesia } \\
\hline Morphine & 51 & 125 & 14 \\
\hline Fentanyl & 45 & 46 & 16 \\
\hline Alfentanil & 96 & 67 & 24 \\
\hline Remifentanil & 43 & 11 & 24 \\
\hline Ketamine & 1 & 3 & 0 \\
\hline
\end{tabular}

Multiple answers were possible. Values are numbers of units. A total of 185 units are included in this analysis. ICU, intensive care unit. 
pilot daily trial sedation practice was not associated with an increased incidence of adverse events.

Sedation withholding is now part of the 'ventilator care bundle', as outlined by the UK Department of Health [33], and recommended by the Surviving Sepsis Campaign [41].

In our survey, $78 \%$ of the ICUs state that they practice daily sedation holding. By comparison, the reported proportion of ICUs practicing sedation withholding in Canada is $40 \%$, in Denmark it is $31 \%$ and in Germany it is 34\% [18-20]. However, our study revealed that only $53 \%$ of ICUs audit their use of daily sedation holding, which suggests that the number of ICUs practicing sedation holding effectively is probably lower than the $78 \%$ stated.

There may be many reasons why ICUs are not practicing daily sedation holding. Devlin and coworkers [42] surveyed American clinicians in 2004 and found that some ICUs were not adopting sedation holding because of a lack of nursing acceptance of this practice, a potential increase in patient selfharm, potential for respiratory compromise and concern about patient comfort.

The high rates of sedation holding reported in this study may reflect increasing awareness and acceptance of the technique and most subsequent studies supporting its safety and benefit.

\section{Choice of agents used}

The sedation guideline published by the ACCM [12] recommends use of fentanyl or morphine for analgesia, midazolam or propofol for short-term sedation, and lorazepam for longer term sedation. The practice in the UK differs greatly from these guidelines. Alfentanil is used more commonly than fentanyl or morphine, likely because of its lesser degree of accumulation and shorter duration of action. For patients expected to require sedation for longer than 24 hours, morphine and midazolam were most frequently chosen, whereas lorazepam is rarely chosen.

Our survey illustrated that the duration of action of the sedating agent was a more important factor in choice of sedating agent than its cost. This concurs with the German sedation survey [20]. It will be interesting to observe whether newer short-acting but more expensive agents (for instance, remifentanil and dexmedetomidine) are chosen for sedation in the future.

\section{Neuromuscular blocking agents}

Muscle relaxing agents are infrequently used in UK ICUs. The few ICUs using neuromuscular blocking agents (NMBAs) in more than $10 \%$ of patients were mostly neurological ICUs. The infrequent use of NMBAs may reflect the increasing emphasis on lighter levels of sedation and the concerns regarding critical illness neuropathy and myopathy [43].

\section{Study limitations}

This study shares the limitations of all surveys in that reporter bias cannot be excluded. Furthermore, only the head of the department was addressed; the answers may therefore only reflect the individual's practice, and may not be representative for the entire unit. Past surveys, however, would have faced similar limitations, and given our good response rate, comparison with surveys in the past and in other countries can be made. The wide range of units responding make it likely that our results reflect actual UK practice appropriately, within the constraints of self-reporting practice.

\section{Conclusion}

An increasing number of ICUs in the UK utilize a sedation guideline and a sedation scoring tool. The Ramsey Sedation Scale is the most frequently chosen assessment score. Sedation holding is done by most but not all of the ICUs. Its implementation compares favourable with that identified in other international sedation surveys. The choice of sedating agent is quite variable and differs from that in other countries. Choice of sedating agent is directed more by duration of action rather by cost. NMBAs are infrequently used outside neurological ICUs.

\section{Key messages}

- The majority of units have a standardized approach to sedation management, using a sedation guideline, sedation scoring and daily sedation holding.

- In contrast to published guidelines and existing evidence, there is still a considerable number of ICUs that do not practice effective daily sedation holding.

- Wide variation exists in the choice of sedating or analgesic agent, with the short-acting opioid alfentanil being a popular choice.

- Only a minority of ICUs use NMBAs regularly.

- Choice of sedating agent is directed more by duration of action rather by cost.

\section{Competing interests}

The authors declare that they have no competing interests.

\section{Authors' contributions}

$H R$ and AK made substantial contributions to the conception, design, analysis and interpretation of the data. MM had substantial involvement in revising and drafting the article, and in interpreting the data. All authors contributed to drafting and revising the article, and approved the final manuscript. 


\section{Additional files}

The following Additional files are available online:

\section{Additional file 1}

A Word document containing the questionnaire sent to all UK ICUs.

See http://www.biomedcentral.com/content/

supplementary/cc7141-S1.doc

\section{Acknowledgements}

We would like to thank Carys Jones, Research Sister in the Royal Berkshire Hospital ICU, for liaising with Intensive Care National Audit \& Research Centre and helping in obtaining the hospital details. We would furthermore like to thank all of the clinicians who participated in this study for filling out and returning the questionnaires. We thank the statistical department of GlaxoSmithKline for help with statistical analysis only.

\section{References}

1. Hansen-Flaschen JH, Brazinsky S, Basile C, Lanken PN: Use of sedating drugs and neuromuscular blocking agents in patients requiring mechanical ventilation for respiratory failure. A national survey. Jama 1991, 266:2870-2875.

2. Roth-Isigkeit A, Brechmann J, Dibbelt L, Sievers HH, Raasch W, Schmucker P: Persistent endocrine stress response in patients undergoing cardiac surgery. J Endocrinol Invest 1998, 21:12-19.

3. Lewis KS, Whipple JK, Michael KA, Quebbeman EJ: Effect of analgesic treatment on the physiological consequences of acute pain. Am J Hosp Pharm 1994, 51:1539-1554.

4. Schelling G: Post-traumatic stress disorder in somatic disease: lessons from critically ill patients. Prog Brain Res 2008, 167:229-237.

5. Burns AM, Shelly MP, Park GR: The use of sedative agents in critically ill patients. Drugs 1992, 43:507-515.

6. Kollef MH, Levy NT, Ahrens TS, Schaiff R, Prentice D, Sherman G: The use of continuous i.v. sedation is associated with prolongation of mechanical ventilation. Chest 1998, 114:541-548.

7. Kress JP, Pohlman AS, O'Connor MF, Hall JB: Daily interruption of sedative infusions in critically ill patients undergoing mechanical ventilation. $N$ Engl J Med 2000, 342:1471-1477.

8. Elliott R, McKinley S, Aitken LM, Hendrikz J: The effect of an algorithm-based sedation guideline on the duration of mechanical ventilation in an Australian intensive care unit. Intensive Care Med 2006, 32:1506-1514.

9. Hogarth DK, Hall J: Management of sedation in mechanically ventilated patients. Curr Opin Crit Care 2004, 10:40-46.

10. Kress JP, Gehlbach B, Lacy M, Pliskin N, Pohlman AS, Hall JB: The long-term psychological effects of daily sedative interruption on critically ill patients. Am J Respir Crit Care Med 2003, 168:1457-1461.

11. Schweickert WD, Gehlbach BK, Pohlman AS, Hall JB, Kress JP: Daily interruption of sedative infusions and complications of critical illness in mechanically ventilated patients. Crit Care Med 2004, 32:1272-1276.

12. Jacobi J, Fraser GL, Coursin DB, Riker RR, Fontaine D, Wittbrodt ET, Chalfin DB, Masica MF, Bjerke HS, Coplin WM, Crippen DW, Fuchs BD, Kelleher RM, Marik PE, Nasraway SA Jr, Murray MJ, Peruzzi WT, Lumb PD: Clinical practice guidelines for the sustained use of sedatives and analgesics in the critically ill adult. Crit Care Med 2002, 30:119-141.

13. Mattia C, Savoia G, Paoletti F, Piazza O, Albanese D, Amantea B, Ambrosio F, Belfiore B, Berti M, Bertini L, Bruno F, Carassiti M, Celleno D, Coluzzi F, Consales G, Costantini A, Cuppini F, De Gaudio RA, Farnia A, Finco G, Gravino E, Guberti A, Laurenzi L, Mangione S, Marano M, Mariconda G, Martorano PP, Mediati R,
Mercieri M, Mondello E, et al:: SIAARTI recommendations for analgo-sedation in intensive care unit. Minerva Anestesiol 2006, 72:769-805.

14. Ostermann ME, Keenan SP, Seiferling RA, Sibbald WJ: Sedation in the intensive care unit: a systematic review. JAMA 2000, 283:1451-1459.

15. Izurieta R, Rabatin JT: Sedation during mechanical ventilation: a systematic review. Crit Care Med 2002, 30:2644-2648.

16. Murray MJ, Cowen J, DeBlock H, Erstad B, Gray AW Jr, Tescher AN, McGee WT, Prielipp RC, Susla G, Jacobi J, Nasraway SA Jr, Lumb PD: Clinical practice guidelines for sustained neuromuscular blockade in the adult critically ill patient. Crit Care Med 2002, 30:142-156.

17. Martin J, Parsch A, Franck M, Wernecke KD, Fischer M, Spies C: Practice of sedation and analgesia in German intensive care units: results of a national survey. Crit Care 2005, 9:R117-R123.

18. Mehta S, Burry L, Fischer S, Martinez-Motta JC, Hallett D, Bowman D, Wong C, Meade MO, Stewart TE, Cook DJ: Canadian survey of the use of sedatives, analgesics, and neuromuscular blocking agents in critically ill patients. Crit Care Med 2006, 34:374-380.

19. Egerod I, Christensen BV, Johansen L: Trends in sedation practices in Danish intensive care units in 2003: a national survey. Intensive Care Med 2006, 32:60-66.

20. Martin J, Franck M, Sigel S, Weiss M, Spies C: Changes in sedation management in German intensive care units between 2002 and 2006: a national follow-up survey. Crit Care 2007, 11:R124.

21. Payen JF, Chanques G, Mantz J, Hercule C, Auriant I, Leguillou JL, Binhas M, Genty C, Rolland C, Bosson JL: Current practices in sedation and analgesia for mechanically ventilated critically ill patients: a prospective multicenter patient-based study. Anesthesiology 2007, 106:687-695. quiz 891-682.

22. Murdoch $S$, Cohen $A$ : Intensive care sedation: a review of current British practice. Intensive Care Med 2000, 26:922-928.

23. Ramsay MA, Savege TM, Simpson BR, Goodwin R: Controlled sedation with alphaxalone-alphadolone. BMJ 1974 , 2:656-659.

24. Riker RR, Fraser GL, Cox PM: Continuous infusion of haloperidol controls agitation in critically ill patients. Crit Care Med 1994, 22:433-440.

25. Riker RR, Picard JT, Fraser GL: Prospective evaluation of the Sedation-Agitation Scale for adult critically ill patients. Crit Care Med 1999, 27:1325-1329.

26. Devlin JW, Boleski G, Mlynarek M, Nerenz DR, Peterson E, Jankowski M, Horst HM, Zarowitz BJ: Motor Activity Assessment Scale: a valid and reliable sedation scale for use with mechanically ventilated patients in an adult surgical intensive care unit. Crit Care Med 1999, 27:1271-1275.

27. de Lemos J, Tweeddale M, Chittock D: Measuring quality of sedation in adult mechanically ventilated critically ill patients. The Vancouver Interaction and Calmness Scale. Sedation Focus Group. J Clin Epidemio/ 2000, 53:908-919.

28. Sessler CN, Gosnell MS, Grap MJ, Brophy GM, O'Neal PV, Keane KA, Tesoro EP, Elswick RK: The Richmond Agitation-Sedation Scale: validity and reliability in adult intensive care unit patients. Am J Respir Crit Care Med 2002, 166:1338-1344.

29. Ely EW, Truman B, Shintani A, Thomason JW, Wheeler AP, Gordon S, Francis J, Speroff T, Gautam S, Margolin R, Sessler CN, Dittus RS, Bernard GR: Monitoring sedation status over time in ICU patients: reliability and validity of the Richmond AgitationSedation Scale (RASS). JAMA 2003, 289:2983-2991.

30. De Jonghe B, Cook D, Appere-De-Vecchi C, Guyatt G, Meade M, Outin $\mathrm{H}$ : Using and understanding sedation scoring systems: a systematic review. Intensive Care Med 2000, 26:275-285.

31. Brook AD, Ahrens TS, Schaiff R, Prentice D, Sherman G, Shannon $\mathrm{W}$, Kollef $\mathrm{MH}$ : Effect of a nursing-implemented sedation protocol on the duration of mechanical ventilation. Crit Care Med 1999, 27:2609-2615.

32. Duane TM, Riblet JL, Golay D, Cole FJ Jr, Weireter LJ Jr, Britt LD: Protocol-driven ventilator management in a trauma intensive care unit population. Arch Surg 2002, 137:1223-1227.

33. Brattebo G, Hofoss D, Flaatten H, Muri AK, Gjerde S, Plsek PE: Effect of a scoring system and protocol for sedation on duration of patients' need for ventilator support in a surgical intensive care unit. $B M J$ 2002, 324:1386-1389. 
34. Adam C, Rosser D, Manji M: Impact of introducing a sedation management guideline in intensive care. Anaesthesia 2006, 61:260-263.

35. Marshall J, Finn CA, Theodore AC: Impact of a clinical pharmacist-enforced intensive care unit sedation protocol on duration of mechanical ventilation and hospital stay. Crit Care Med 2008, 36:427-433.

36. Bucknall TK, Manias E, Presneill JJ: A randomized trial of protocol-directed sedation management for mechanical ventilation in an Australian intensive care unit. Crit Care Med 2008, 36:1444-1450.

37. Krishnan JA, Moore D, Robeson C, Rand CS, Fessler HE: A prospective, controlled trial of a protocol-based strategy to discontinue mechanical ventilation. Am J Respir Crit Care Med 2004, 169:673-678.

38. Kress JP, Vinayak AG, Levitt J, Schweickert WD, Gehlbach BK, Zimmerman F, Pohlman AS, Hall JB: Daily sedative interruption in mechanically ventilated patients at risk for coronary artery disease. Crit Care Med 2007, 35:365-371.

39. de Wit M, Gennings C, Jenvey WI, Epstein SK: Randomized trial comparing daily interruption of sedation and nursing-implemented sedation algorithm in medical intensive care unit patients. Crit Care 2008, 12:R70.

40. Mehta S, Burry L, Martinez-Motta JC, Stewart TE, Hallett D, McDonald E, Clarke F, Macdonald R, Granton J, Matte A, Wong C, Suri A, Cook DJ: A randomized trial of daily awakening in critically ill patients managed with a sedation protocol: a pilot trial. Crit Care Med 2008, 36:2092-2099.

41. Dellinger RP, Levy MM, Carlet JM, Bion J, Parker MM, Jaeschke R, Reinhart K, Angus DC, Brun-Buisson C, Beale R, Calandra T, Dhainaut JF, Gerlach H, Harvey M, Marini JJ, Marshall J, Ranieri M, Ramsay G, Sevransky J, Thompson BT, Townsend S, Vender JS, Zimmerman JL, Vincent JL: Surviving Sepsis Campaign: international guidelines for management of severe sepsis and septic shock: 2008. Intensive Care Med 2008, 34:17-60.

42. Devlin JW, Tanios MA, Epstein SK: Intensive care unit sedation: waking up clinicians to the gap between research and practice. Crit Care Med 2006, 34:556-557.

43. Hund E: Myopathy in critically ill patients. Crit Care Med 1999, 27:2544-2547. 